\author{
Giuseppe Andrea Liberti* \\ Università degli Studi di Napoli "Federico II"
}

\title{
MICHELE SOVENTE E LE PAROLE DELLA SCIENZA. SCHEDE SUL LESSICO DI CONTROPAR(AB)OLA
}

\begin{abstract}
Dopo una breve ricognizione della presenza e del ruolo del linguaggio scientifico nella poesia italiana contemporanea, il contributo indaga un caso specifico di uso del lessico medico-scientifico in una raccolta poetica. Michele Sovente (1948-2011), conosciuto come autore "trilingue" che adopera l'italiano, il latino e il dialetto cappellese nella sua scrittura, comincia il suo percorso poetico usando esclusivamente l'italiano. Ciò detto, i primi libri di Sovente presentano numerosi termini provenienti da vari campi scientifici che testimoniano un interesse per la commistione linguistica. Tale vocabolario tecnico risulta particolarmente presente in Contropar(ab)ola, il secondo libro dell'autore, nel quale il lettore può leggere parole come 'gerovital', 'gengivite' o 'emottisi', ma anche termini della chimica ('nitrile') o della biologia ('ozono', 'plantigradi'). Lo studio mira a chiarire le modalità d'impiego del lessico scientifico, adottato in chiave metaforica o nel rispetto dei suoi effettivi significati, al fine di mostrare come la terminologia scientifica possa essere utilizzata in contesti tematici diversi.
\end{abstract}

Parole chiave: Michele Sovente, poesia, scienza, lingua poetica, sperimentalismo, Novecento.

La storia delle coincidenze, sovrapposizioni e reciproche influenze tra il linguaggio scientifico e quello letterario è antica, e ricostruire i rispettivi processi di formazione condurrebbe innanzitutto a riconoscere una scissione tutto sommato tardiva tra scienze dure e saperi umanistici. Chi volesse soffermarsi sulle modalità di messa a punto dei linguaggi tecnico-scientifici, nome fin troppo generalista che racchiude branche anche diversissime della cultura scientifica, dovrebbe però interrogarsi sul rapporto che un lessico specialistico intrattiene con la lingua quotidiana. È proprio da quella, in effetti, che germinano i significanti della scienza, che solo attraverso processi di predeterminazione e ridefinizione del significato acquisiscono gradualmente una propria autonomia tecnica. Così nacque, per fare subito un esempio

*giuseppeandrea.liberti@unina.it 
di particolare rilievo, il vocabolario di Galileo Galilei, che rifiutava sì la terminologia di una cultura opposta e anzi ostile alla scienza quale quella scolastico-aristotelica, ma neppure accoglieva il lessico di manovali e "vili meccanici", che non avrebbe valorizzato il portato delle sue scoperte essendo tecnico ma ancora eminentemente pratico, non già in grado di farsi teorico (cfr. Porro 1973: 185-187). Col tempo, e soprattutto col progredire delle ricerche scientifiche, è venuto formandosi un linguaggio settoriale riconoscibile, fatto tanto di precipitati lessicali ormai immediatamente avvertibili nel loro significato scientifico per quanto provenienti dalla lingua comune, quanto di innovazioni che ne esaltano la specificità.

Dobbiamo agli storici della lingua l'individuazione dei tratti salienti di questa branca dell'italiano ${ }^{1}$; ma è opportuno ricordare - per quanto possa sembrare scontato - che il linguaggio scientifico è a tutti gli effetti una lingua globale, e le tendenze lessicali che possiamo riscontrare nei testi di medicina o chimica nostrani saranno più o meno le stesse nella letteratura scientifica del Canada, della Russia o del Maghreb. Prima sua caratteristica, come si è già accennato, è la rideterminazione semantica che rende convenzionali termini comuni, che può coinvolgere tanto espressioni della lingua quotidiana quanto tecnicismi provenienti da altre branche scientifiche: basti qui il caso di 'resistenza', che in fisica indica qualunque forza che si opponga a una forza agente, mentre in biologia vale "il complesso dei caratteri meccanici di un tessuto o di un organo, legati alla coesione interna dei materiali che lo costituiscono" ('resistenza', in NDMSL 1976: 934). La lingua comune funge da serbatoio di tecnicismi collaterali (cfr. Serianni 1989: 105), vale a dire espressioni stereotipiche preferite per la sfumatura tecnica che riescono ad addurre; il pericolo di sinonimie o sovrapposizioni di significati è scongiurato dalla monoreferenzialità del lessico scientifico, organizzato come una nomenclatura in cui ciascun termine assume una definizione concettuale precisa all'interno di una tassonomia gerarchica.

Inevitabilmente, il progresso necessita di innovazioni concettuali e pertanto terminologiche; al saccheggio del vocabolario comune, la lingua della scienza aggrega battesimi lessicali e arricchimenti per mezzo di segni aggiuntivi, al fine di soddisfare i bisogni di denominazione propri di un particolare settore. Avremo quindi particelle maggiormente ricorrenti in un'area scientifica piuttosto che in un'altra, o veri e propri simboli, integrati nella terminologia, che connotano solo un determinato campo di studi. Importantissimo è poi, nell'ambito dei neologismi, l'ampio uso di prefissoidi e suffissoidi generalmente ripresi dal latino e soprattutto dal greco (uso che potremmo far rientrare tra i segni aggiuntivi citati poc'anzi), per non

${ }^{1}$ Il presente studio segue e riprende le indicazioni offerte da Altieri Biagi (1990), Mengaldo (1994: 37-43), Porro (1973) e Zublena (2002). 
parlare delle neo-formazioni costituite da termini delle lingue antiche. Una spigolatura nel Saie/Larousse propone il 'diachilon', un impiastro cicatrizzante e agglutinante il cui nome è formato dalle parole greche diá (con) e chilòn (succo; cfr. NDMSL 1976: 296). Infine, è ancora tipico della scienza il ricorso alle sigle. Si pensi solo ai tantissimi DNA, RNA, IgM o Rb della medicina e della biologia, a sigle chimiche di tipologia affine come PVC, che altro non è che il polivinilcloruro, oppure ai simboli degli elementi chimici che, addirittura, traggono le loro forme dalle denominazioni latine e greche (è il caso di Au, simbolo dell'oro, da 'aurum'). Abbreviazioni che nascono per agevolare l'analisi, ma che finiscono col surclassare in termini di riconoscibilità la forma estesa.

Quelle enumerate sono solo alcune delle caratteristiche della semantica scientifica, più o meno verificabili leggendo qualsiasi saggio dedicato a conoscenze non umanistiche $\mathrm{o}$, e contrario, opere letterarie in grado di introiettare lingue, concetti e strutture da campi disparati - ed è scontato dire che il riferimento principale è alla forma costitutivamente ibrida del romanzo. Eppure, in questa sede non si intende parlare di prosa, né di quella, godibilissima, dei trattati di Galilei, né dei congegni linguistici di Gadda o Volponi. Sarà invece la poesia l'obiettivo unico della nostra indagine, che con la scienza forma un binomio saldatosi agli albori della cultura occidentale, come testimoniano i numerosi Perí phýseos in versi della filosofia greca o il fondamentale (e destinato a tornare nella nostra trattazione) De rerum natura di Tito Lucrezio Caro. Il presente lavoro si occupa di anni assai più recenti, più specificamente del secondo e tardo Novecento, un'epoca durante la quale la poesia si è mostrata particolarmente sensibile ai cortocircuiti tra codici linguistici, favoriti dai processi di contaminazione dei linguaggi risalenti per lo meno a un secolo prima.

Le prime e più significative manifestazioni di tali processi, pur preminenti nella poesia del XX secolo, vanno collocate nel secondo Ottocento, quando sia poeti minori come Emilio Praga o Vittorio Betteloni, sia grandi autori come Leopardi, Carducci e soprattutto Pascoli contribuiscono a introdurre nel corpo lirico specialismi e tecnicismi, tratti dai glossari di diverse branche del sapere ${ }^{2}$. Già nel Seicento, in vero, si era assistito a un utilizzo di termini inusuali per la grammatica lirica, e la poesia scientifica del Settecento aveva fatto largo uso del lessico appropriato. Nell'Ottocento, tuttavia, l'immissione di tecnicismi si accompagna a più generali innovazioni in ambito metrico e grammaticale senza le quali la poesia novecentesca sarebbe stata semplicemente impossibile (cfr. Bozzola 2016: 141). Basti il

${ }^{2}$ Un profilo sintetico del rapporto tra lingua scientifica e lingua poetica è delineato in Ortore (2016: 171-176). Sul tardo Ottocento si vedano in particolare Bozzola (2014, 2016). 
caso di Pascoli: con lui, ideologicamente legato alla stagione positivista, si verifica l'ingresso massiccio di termini della zoologia e della botanica, che servono a connotare le 'cose' per come sono. Fino ad allora, non era pensabile trovare, in un testo lirico, nomi di piante e fiori come la 'digitale purpurea', ma neppure sarebbe stato agevole rintracciare gli apparentemente più comuni 'melo', 'mandorlo' o le 'tamerici'. Non è ancora, quello pascoliano, un lessico compiutamente scientifico, visto che molti dei nomi adoperati sono desunti dall'uso popolare e dalle parlate locali; la volontà di precisione e determinatezza che è alla base delle sue scelte, però, apre già la lingua poetica al vocabolario scientifico, come ha riconosciuto un critico sensibile al tema quale Luciano Anceschi (1976: 49):

Il Pascoli opera sull'oggetto con infiniti accorgimenti per renderlo più particolare, più evidente $\mathrm{e}$, perciò, secondo la sua idea, più poeticamente efficace [...]. Lo colloca tra altri oggetti - fino a sfiorare l'elenco - in modo da evocare un ambiente significativo e già denso di atmosfera, in cui l'oggetto emerge in modo particolarissimo. E si giova di tutta una nomenclatura popolare [...] o scientifica o tecnica - e cerca suoni studiati fino a giungere all'onomatopea: in ogni modo una musica facile - direi una melodia - consente all'oggetto un rilievo, una preminenza significativa. [...] Sono gli oggetti di un uomo fiducioso nella scienza, positivista a suo modo, fino ad augurare una poesia della scienza, una poesia che faccia lievitare il certo e, nello stesso tempo, visionario e dotato di una particolare disposizione per esplorare (in modo interiore) l'inconoscibile...

Non è necessario stabilire se il lessico introdotto dal poeta di San Mauro abbia avuto o meno successo nella storia della lingua poetica italiana; conta invece che con Pascoli la lirica esca definitivamente dalla tirannia dei poetismi, si apra al linguaggio tecnico e introduca termini prima del tutto estranei al corpus storicamente impostosi. Se, in definitiva, Eugenio Montale può aprire e chiudere i suoi Ossi di seppia citando i 'ligustri' e 1' 'erbaspada', è anche in virtù del lascito pascoliano.

Negli anni, e in un contesto di più generale rimodulazione formale e contenutistica della poesia ${ }^{4}$, i vocabolari degli autori novecenteschi si sono sempre più arricchiti di queste immissioni, anche se la scelta di adottare un termine tecnico piuttosto che un altro e la funzione dello stesso nel discorso poetico varia sensibilmente a seconda degli orientamenti ideologici

${ }^{3}$ Cfr. Montale 2013: "Ascoltami, i poeti laureati / si muovono soltanto fra le piante / dai nomi poco usati: bossi ligustri o acanti" (I limoni, 12, vv. 1-3); "Riviere, / bastano pochi stocchi d'erbaspada / penduli da un ciglione / sul delirio del mare [...]" (Riviere, 256, vv. 1-4).

${ }^{4}$ L'assieme delle innovazioni linguistiche, metriche e di contenuto operate sulla poesia italiana ha portato qualcuno a parlare di una fase compiutamente 'post-lirica': cfr. Testa (2005) e Zublena (2014). 
e stilistici dell'autore. Un 'riduttivo uso espressionistico e negativo' in cui il lessico scientifico "è spesso mero sinonimo di alienazione o di dominio della tecnica" (Ortore 2012: 112) è solitamente proprio, per esempio, dei Novissimi e più in generale degli autori delle nuove avanguardie, il che ovviamente non esclude che possano esservi eccezioni o altre posizioni - in un raggruppamento, per altro, già di per sé assai poco omogeneo. Tra i più genuinamente interessati all'apporto (non solo) linguistico che la scienza può dare alla poesia figura Elio Pagliarani, che nel volume dall'eloquente titolo Lezione di fisica e Fecaloro assembla versi in cui compaiono il lessico, la sintassi e addirittura - grazie alle tecniche del cut-up e del ready-made - la forma del trattato scientifico 5 .

Sebbene risultino più evidenti nella poesia novissima, prestiti e contaminazioni con la lingua della scienza sono comuni a buona parte dei maggiori poeti del secondo Novecento, persino quelli più insospettabili come Attilio Bertolucci, il cui linguaggio quotidiano ma a forte vocazione pittorica sa comunque accogliere parole come 'subfebbrile' (cfr. Mengaldo 1994: 237238). Ma si pensi anche al caso di Giovanni Giudici, che annette materiali linguistici disparati sin dalle sue prime prove. Se nella prima fase della sua scrittura è il lessico burocratico-giuridico a prevalere, sono poi i termini di area medico-anatomica e biologica a segnare le ultime raccolte, con qualche significativa immissione di lessemi astronomici e chimici ${ }^{6}$. Traggo qualche esempio particolarmente eloquente dalla schedatura di Testa. Dai due volumi di Poesie 1953-1990 (1991): 'anamnesi', 'coccige', 'ecolalia', 'embolo', 'emiplegia', 'fanerogamiche', 'focomelici', 'magnetostrittiva'; da Quanto spera di campare Giovanni (1993): 'miceti', 'polinomio' e ‘table'; da Empie stelle (1996), infine, 'eclampsìa' ed 'encefalo'. Nel caso di autori distanti dalle tante avanguardie del Novecento, le presenze scientifiche rispondono a esigenze di precisione semantica che, per essere soddisfatte, non possono fare a meno di parole altamente connotate.

${ }^{5}$ Si veda Fecamore di Elio Pagliarani: "Quando comincia a svilupparsi un deserto / quando la vegetazione naturale originaria soccombe gradualmente sotto i colpi della siccità / della mancanza di rugiada mattutina dell'inaridimento progressivo della terra, la vita continua / a combattere, sorge un nuovo tipo di vita, una vegetazione secondaria che si adatta / alle dure condizioni di esistenza del deserto emozionale: il gambo del cactus consiste / di fibre isolate, la pianta desertica sviluppa foglie cuoiose e pungenti la struttura clorofillica / è limitata all'estremità dei rami è adattata all'estrema scarsità d'acqua" (Pagliarani 2006: 222).

${ }^{6}$ Un dettagliato studio sulla lingua di Giudici è Testa (1999: 111-133); per le osservazioni sugli scientismi, si vedano le pagine 118-119 (da cui traggo anche gli esempi successivi). 
Arrivando agli ultimi decenni del secolo breve, non meno traboccanti di prelievi lessicali che, anzi, assurgono in tutto e per tutto a tratto caratterizzante della lingua poetica, si incrocia una strana esperienza 'trilingue', in cui prende corpo una sfida dialettica tra significanti di epoche distanti e provenienze coincidenti. $\mathrm{Ci}$ si perdonerà il tono misterioso, che ben si addice a un'introduzione a Michele Sovente, il poeta flegreo attivo tra gli anni Settanta e i primi lustri del XXI secolo noto per le sue raccolte in cui lo stesso testo viene riscritto in italiano, latino e dialetto cappellese ${ }^{7}$. La triangolazione linguistica lascia scaturire suggestioni diverse da un medesimo argomento, che nel caso in questione può andare dall'indagine della dimensione ctonia e delle figure invisibili che animano lo spazio flegreo, la decodifica dei messaggi che emergono dai resti del passato, o ancora l'analisi del legame tra natura e parola poetica. Sono alcuni dei nuclei tematici che ossessionano Sovente, e che trovano il miglior mezzo di approfondimento nell'uso di tre lingue su un piano di assoluta parità di valore, ognuna in grado di porre l'accento su un tratto peculiare dell'oggetto in esame ${ }^{8}$. Bisogna dire che Sovente comincia ad adoperare il latino solo a partire dal suo terzo libro, il poemetto Per specula aenigmatis (1990), mentre il dialetto subentra nel quarto, Cumae (1998), dopo alcune sporadiche comparse in rivista. Le prime due raccolte sono esclusivamente in lingua italiana, e forse per questo non hanno attirato l'attenzione dei critici e dei lettori. In realtà, questi libri, L'uomo al naturale e Contropar(ab)ola, rispettivamente del 1978 e 1981, presentano interessanti soluzioni sperimentali, in linea con tante tendenze della maggiore poesia italiana pubblicata tra anni Settanta e primi anni Ottanta. Sovente, di formazione prettamente umanistica ma presto interessatosi a una disciplina di confine come l'antropologia culturale, che insegnò all'Accademia di Belle Arti di Napoli, non mancò di interessarsi alle possibilità apportate dall'ampliamento del linguaggio all'espressività e alla potenza comunicativa del medium poetico. Il linguaggio scientifico entra in tensione con una poesia in cui il gioco verbale si interseca spesso con la persistente prospettiva politica e una nuova, emergente presenza dei

${ }^{7}$ Nato a Cappella, nei Campi Flegrei, nel 1948, Michele Sovente è stato poeta e docente di Antropologia culturale all'Accademia di Belle Arti di Napoli. Tra i suoi libri si ricordano L'uomo al naturale (1978), Contropar(ab)ola (1981), Per specula aenigmatis (1990), Cumae (1998), Carbones (2002), Carta e formiche (2005), Bradisismo (2008) e Supersiti (2009). Su Michele Sovente, sono fondamentali gli studi raccolti nel fascicolo monografico di Istmi (2013); si vedano inoltre Alfano (2010), De Blasi (2003), Grasso (2012), e sia consentito rinviare a Liberti (2018) e all'edizione critica e commentata della quarta raccolta di Sovente, Cumae (cfr. Sovente 2019).

${ }^{8} \mathrm{Si}$ noti che negli ultimi libri di Sovente, e specificamente in Bradisismo (2008) e Superstiti (2009), fa capolino una quarta lingua poetica, il francese. 
temi del privato. Il presente studio si gioverebbe non poco di studi organici sulle prime raccolte dell'autore, che mancano invece di indagini accurate, ma se si sceglie di trattare un aspetto a prima vista marginale dell'esperienza linguistica di Sovente è al fine di cominciare una verifica di quanto della prima stagione torni nella produzione bi- e trilingue.

Ci occuperemo, in particolare, del secondo libro di Sovente, Contropar (ab)ola ${ }^{9}$, perché quello in cui maggiore è la presenza del lessico tecnico-scientifico, ma rimarrà necessario fare qualche riferimento agli altri capitoli dell'opus soventiano per capire come e fino a che punto queste immissioni segnino la produzione del Nostro. È il 1981: Sovente ha 33 anni, è già docente presso l'Accademia di Napoli, e ha pubblicato, sempre per i tipi di Enrico Vallecchi, un libro dalle forti venature politiche come L'uomo al naturale. La nuova raccolta rappresenta l'attraversamento dei fermenti che animano l'ambiente culturale napoletano degli anni Settanta, il cui grado di autocoscienza e formalizzazione degli espedienti di eversione del fatto poetico è, pur tenendo presenti le differenze tra percorsi di ricerca, tra i più alti in Italia. Contropar ( $a b$ )ola esibisce una libertà metrica decisamente più spiccata rispetto alle successive prove poetiche, così come dal punto di vista linguistico non è assimilabile ai corpora di parole semanticamente affini delle stesse. Tuttavia, sembra di poter dire che proprio negli scientismi si possano intravedere i prodromi di alcune costanti tematiche e figurali della produzione co-linguistica. Vedremo più avanti in che modo il lessico scientifico indirizzi verso la grande poesia degli anni Novanta-Duemila; prima, è necessario vedere nel concreto di quali parole si sta discutendo.

Tra gli ambiti scientifici che maggiormente influenzano il lessico di Contropar(ab)ola spicca quello clinico, quindi la medicina, la farmaceutica e 1'anatomia. Non dovrebbe stupire che proprio quello medico sia il campo più saccheggiato, quello dal quale maggiori sono i prelievi lessicali: il linguaggio medico è il meno scientifico dei linguaggi scientifici, perché la medicina è la scienza che viene maggiormente a contatto con l'uomo comune, ed è forse la sola scienza che ha un "pubblico-oggetto" e non solo "soggetto [...] cioè coincidente con la massa di chi la pratica" (Mengaldo 1994: 43). Un poeta come Sovente, che mai, persino nelle sue sperimentazioni e commutazioni linguistiche più ardite, perde di vista la comprensibilità della sua scrittura, ricorre al termine medico senza temere di risultare eccessivamente incomprensibile, perché quel tipo di vocabolario risponde a esigenze diffuse. Del resto, a terminologie meno familiari come 'anamnesi' (Anamnesi o agnizioni,

${ }^{9}$ Le citazioni da Contropar(ab)ola sono tratte dalla prima e finora unica edizione del volume, del 1981. I titoli delle poesie a cui si fa riferimento sono indicati tra parentesi e seguiti dal numero di pagina; dalle seconde citazioni si riporta invece il solo titolo, salvo nei casi in cui la citazione supera i tre versi. 
11), 'metastasi' o 'leucociti', altro nome dei globuli bianchi (Meta, 76) corrispondono sostantivi di largo utilizzo come 'virus', 'vaccino' (Emergenza, 33), 'febbre' (Disinganno, 57) o 'battèri' (Gre, 74).

Nella poesia di Contropar(ab)ola, sono proprio le parole mediche e anatomiche a essere maggiormente utilizzate nel loro significato propriamente tecnico. Si osservi, per esempio, la presenza delle malattie in questa poesia. Quando Sovente ricorre ai nomi di malattia o patologia, non ne fa un uso figurato; 'emottisi' indica proprio l'uscita del sangue dalla bocca, come accade ne L'orditura ('che fino a ieri crepava di salute / che sta in preda alla magrezza all'emottisi'; 12). Altre patologie del libro sono la 'gengivite' (Folle novembre: secolo folle, 45), la 'febbre' (definita 'luminescente'; Disinganno, 57), la 'flebite' che esce dalle mani del direttore d'azienda (Un vuoto eccitante, 58), lo stato di 'catalessi' e, ancora, 1' 'ebetudine catarrale' (Cata, 71-72). Identica esattezza lessicale si verifica quando Sovente deve riferirsi ai farmaci: 'gerovital' (Sopra un muro, 32), 'aspirine' (Un vuoto eccitante, 58) e 'cardiotonico' (Folle novembre: secolo folle, 45) sono nomi precisi - i primi due sono addirittura marchi registrati. Largo spazio viene poi concesso al corpo, ma l'anatomia di Contropar(ab)ola è decisamente distante tanto da quella petrarchesca quanto, in verità, dalle forme novecentesche di attenzione alla corporeità. Ben al di là delle 'mani' e dei 'polsi', o dei più spinti 'seni', si incappa qui in 'addomi' (Di giorno, di notte, 47), 'tonsille' (Bianco su bianco, 62), in una 'laringe' (Pur si compi, 97). Diverse componenti anatomiche vengono indicate con la forma, scientificamente esatta, 'nome + aggettivo in -ale': 'taglio ombelicale' (La macchina esatta, 29); 'sacche intestinali' (Dopo il naufragio, 39); 'midollo spinale' (Bianco su bianco); 'umido vaginale' (L'ipotesi, 84).

Altrettanto fortunati sono i prelievi dagli ambiti della fisica e soprattutto della chimica, che offrono un ampio ventaglio di nomi e oggetti utili ad ampliare lo spazio poetico. Non è un caso che la maggior parte di questi siano nomi di elementi chimici, allotropi e composti: 'fluoro' (Sopra un muro); 'ossigeno', 'nitrile' (Senza risposta, 37); 'ozono' (Il boia, 48); 'anidride solforosa' (Tu sei il mio re!, 53); 'polistirolo' (L'artigiano impagato, 54); il chimismo arcaizzante 'fuocogreco' (Gre, 74); 'carbonfossile' (Meta); 'plexiglass' (Equazioni impossibili, 89); 'clorati' e l'aggettivo 'aurifere' (Nel vortice, 92). Dalla fisica nucleare provengono invece 'atomo' (Senza risposta) e parole correlate - poiché pertinenti alla manipolazione e all'evoluzione degli stessi - come 'radiattive' (Il gatto, 21) e 'centrali nucleari' (La rosa: la cosa, 51).

A questi due grandi raggruppamenti, si affiancano termini desunti dal mondo dell'ingegneria e dell'elettronica, dall'astronomia ed elementi di lessico matematico-geometrico. Se ne offrono alcuni campioni: 
MATEMATICA, GEOMETRIA: 'parallelepipedo' (Geometria e dialettica, 52), 'algebra' (Tu sei il mio re!), 'equazioni' (Equazioni impossibili). 'asimmetrie' (Pur si compi, 97), 'multipli' (Nel moto ondoso, 100), 'sinusoide' (Il pugile bambino, 103);

INGEGNERIA, INDUSTRIA, ELETTRONICA: 'cablogramma' (Dopo il naufragio), 'intelaiatura' (L'artigiano impagato), 'hangar' (Cronaca di un viaggio, 61), 'tubo catodico' (Cata, 71), 'radar' (Nel vortice);

BOtANicA, zoologia: 'madreporiche' (Il vecchio guru, 22), 'plantigradi' $(A b, 70)$, 'coleotteri' (Il grande stomaco, 90), 'papaveracei' (Pur si compi);

astronomia: 'ufo' (Disinganno), 'meteora' (Da quale parte?, 87);

PSICOLOGIA: 'abulia' $(A b, 69)$.

La compilazione effettuata dimostra quanto presente sia la lingua della scienza - o meglio, di scienze anche molto distanti tra loro - nella Contropar(ab)ola di Sovente. Resta da capire perché il tutto non si riduca a una muta carrellata di parole, come queste presenze si pongano nei confronti dell'uso di espressioni scientifiche in poesia, come rientrino in un progetto poetico ben definito e, quindi, che ruolo svolgano nella successiva produzione dell'autore. È giusto riconoscere che Sovente fa spesso uso della 'funzione espressionistica' della lingua scientifica, senza che questa si elevi dallo statuto di tecnicismo iper-connotato piazzato al fine di provocare il lettore poco avvezzo al termine allotrio. I dettagli anatomici svolgono spesso il ruolo di rafforzativi espressivi; e così, il colore bianco non solo penetra gli occhi del poeta, ma lo trafigge 'fin dentro al midollo spinale fin dentro alle / sporche tonsille fin dentro al...' (Bianco su bianco; corsivi nostri). Il bianco raggiunge quindi le cavità profonde dell'uomo, che ne coordinano la sensibilità attraverso il sistema nervoso (il midollo spinale) e lo espongono alle difficoltà respiratorie prodotte dal colore 'OSSESSIVO' e, aggiungeremmo noi, mozzafiato. Le tonsille sono non a caso qualificate come 'sporche', per rendere a un tempo più cruda l'immagine e credibile l'effetto fisico provocato dal bianco, poco incisivo in presenza di semplici 'tonsille' che svolgono, quando in buone condizioni, funzione protettiva. Sovente è attento a non lasciare nulla al caso: sa che, in poesia, ogni parola si colora di ambiguità, ma allo stesso tempo porta con sé un bagaglio di significati esatti che, nel caso della terminologia scientifica, diventano addirittura ineludibili. Anche nei casi in cui si accostino elementi di scienze diverse, è cura del poeta scegliere concetti che riescano a legarsi tra loro. Un verso di Meta recita "per metastasi l'amore si è volto in carbonfossile". Qui abbiamo, propriamente, la trattazione del tema erotico attraverso prelievi dai campi semantici della medicina e della chimica; ma sebbene prevalga l'uso espressionistico-retorico, non di meno la scelta di simili espressioni denuncia una volontà di precisione impossibile da aggirare con alternative dai medesimi ambiti. Possiamo interpretare il verso in questo modo: il profondo e reiterato dolore rappresentato dalle metastasi trasforma l'amore in 
un oggetto duro, nero e poco avvenente, eppure in grado di generare calore (il carbonfossile). Il linguaggio aleatorio del sentimento viene tradotto in due immagini assolutamente fisiche, avvicinate dal loro essere prodotti di mutazioni.

Abbastanza prevedibile, ancora, il ricorso in chiave quasi esclusivamente metaforica alla terminologia matematico-geometrica, visto che le scienze dell'astrazione si rivelano un arsenale di figure su cui modellare similitudini e metafore. Ecco dunque le 'equazioni' che dovrebbero stabilire uguaglianze impossibili (Equazioni impossibili), la 'sinusoide' che descrive l'andamento ondulato dei pugni del pugile (Il pugile bambino), fino alla definizione di un soggetto che è "coriacea figura che va dal rosso all' azzurro / misura e mistura di asimmetrie e infinito" (Pur si compi; corsivo nostro). E nonostante tutto, resta la grande attenzione riservata alla scelta dei concetti destinati agli autentici messaggi di questa poesia. Sovente cita spesso i settori della scienza, scegliendo ogni volta con attenzione il discorso scientifico che possa esprimere un preciso aspetto dei temi sociali, intimi o esistenziali:

l'essenziale è convincersi di avere fino in fondo

fatto il proprio dovere di comparse dignitose che hanno memorizzato fino in fondo la biologia della propria miseria o della propria rovinosa ricchezza [...] (Finiremo, 64; corsivo nostro).

'Biologia', dunque studio delle leggi che governano l'iter della 'miseria' e della 'ricchezza'; la sorte delle "comparse dignitose" si compone di fenomeni osservabili come nel regno naturale, in quanto inerenti alla loro vita, bios. Calcoli precisi e matematiche certezze governano invece il mondo del capitale:

In perdita al tatto i giorni dell'amore facce a migliaia in smorfie contratte ho saldato i debiti ma l'algebra dei padroni poco mi aiuta a tenere il conto era bello tenersi per mano giocare con cigni d'aria (Tu sei il mio re!, 53; corsivo nostro).

È interessante che Sovente non faccia riferimento all'economia, la branca del sapere che analizza la produzione di ricchezza ma anche i rapporti sociali a essa inerenti, prediligendo la fredda esattezza dei conti padronali ("l'algebra dei padroni"). Proprio quando ricorre alle nozioni generali, Sovente muove forme di critica ai saperi scientifici, insufficienti a esaurire la complessità del mondo reale e di ogni giorno: 
consulto le mie altezzose nozioni di geometria

e di stracciata socioantropologia per far

quadrare

il vuoto tra una piastrella e l'altra del bagno

e un cadavere che giace lì da mesi

(Geometria e dialettica, 52; corsivi nostri).

Ancora una volta, una scelta in linea con le figure testuali, in cui le scienze forniscono strumenti utili a 'far quadrare' le cose (la 'geometria' le piastrelle, la 'socioantropologia' un corpo defunto) e allo stesso tempo devono accompagnarsi a una non-scienza essenziale quale la dialettica.

Fin qui, si direbbe, una consonanza con lo 'spirito del tempo': 1'acuminatezza semantica avvicina Sovente alla larga schiera di poeti novecenteschi che, discepoli dell'avanguardia o meno, avvertono come anacronistico il tener fuori dallo spazio poetico i codici linguistici delle scienze, che non hanno ormai più alcun motivo per non contaminarsi (e contaminare) con i plurimi registri della versificazione del XX secolo. Nella sua stagione di maggior tensione 'sperimentale', che si esprime nell'adozione di strategie linguistico-formali proprie di tutto il campo avverso alla cosiddetta 'parola innamorata', l'autore flegreo sfrutta le possibilità di commistione tra strutture poetiche e linguaggi scientifici, saggiando la loro adattabilità alle esigenze della lirica e inserendoli nel suo vocabolario; più originale sarà, piuttosto, assistere all'evoluzione dei prelievi nella successiva produzione soventiana. In effetti, non avrebbe molto senso analizzare Contropar( $a b$ )ola come caso unico di presenze del lessico scientifico nella poesia del Flegreo, e il nostro discorso potrebbe agevolmente interrompersi qui, con una sommaria analisi dei vocaboli innestati nel corpo della (post-)lirica; ma un esperimento condotto sulla lingua dell' autore costringe ad allargare la visuale, risultando difficile credere che un simile sforzo di ricerca linguistica si interrompa dopo una sola prova ${ }^{10}$.

È del resto da osservare il duplice senso che assume la componente scientifica, che passa da un uso espressionistico a una funzione lirico-didascalica. Michele Ortore ha riconosciuto l'esistenza di una 'via lucreziana alla lirica' che fa a meno dei retaggi romantici e decadenti per affidarsi a uno stile anticlassico, in cui i lessemi assumono significato tanto sul piano poetico quanto su quello scientifico (cfr. Ortore 2016: 176-180; e si veda anche Id. 2012: 112). Non di rado queste due prospettive agiscono in sinergia, attivando sì la connotazione negativa o per lo meno critica cara all'avan-

${ }^{10}$ Ciò non significa che l'opus di Sovente vada letto come un unico 'romanzo in versi', ed è anzi possibile rintracciare espedienti tematici e figurali (oltre che, ovviamente, inerenti alla graduale introduzione delle altre lingue) utili all'individuazione di sequenze e 'capitoli' interni alla sua bibliografia. 
guardia, politicamente impegnata a combattere la tecnocrazia ab-usando delle sue parole, ma senza eliminare dall'orizzonte poetico un campionario di espressioni che contribuisce ad aprire la lirica a campi del sapere non necessariamente ostili o inconciliabili con essa. Se il rischio, come osserva ancora Ortore, è che in tanta produzione in versi possa "non avere senso chiedersi che significa un tecnicismo" (Ortore 2016: 178), i rilievi finora effettuati su Contropar(ab)ola dimostrano che nessun termine della raccolta è scelto allo scopo di épater les lecteurs, bensì per veicolare una precisa immagine, anche se di non immediata decifrazione.

Non solo. È nostra opinione che il 'lucrezianesimo' di Sovente trovi almeno un'altra forma d'espressione nei suoi libri posteriori. Formatosi nella temperie della poesia di ricerca napoletana, egli trova presto un suo linguaggio e una sua dimensione espressiva in cui le acquisizioni di gioventù si armonizzano, restituendo motivi e dettagli fortemente personali che concorrono alla costruzione di un immaginario ben definito. Già con $\mathrm{Per}$ specula aenigmatis $(1990)^{11}$, prima poderosa prova bilingue di Sovente, la direttrice scientifica si stempera nel caos baroccheggiante del poemetto; eppure, benché si confondano con gli ossessivi giochi fonici e le reiterate combinazioni semantiche, sono ancora riconoscibili le 'cellule' (85), geologismi come 'ametista' (87), 'topazio' (111) o 'smeraldo' (119), fino a costruzioni più complesse come 'ruota transorbitale' (85). L'universo di Cumae si basa invece su un dualismo grande-piccolo perfettamente espresso da termini di provenienza scientifica come 'pianeti' e le solite 'cellule' (I pianeti roventi del buio, 39) e 'atomi', 'galassie' e 'pulviscoli' (Prato e naufragio, 123), 'nebulose' (C'è un brusio, 21) e 'particelle' (Definizioni, 81). È un tipo di immaginario che trova qualche anticipazione proprio in Contropar(ab)ola. Si osservi l'esordio di Nel vortice (92), nel quale si riconoscono

le fiancate il mogano le altezze i riquadri

clorati lucidati

che bianca e poi più bianca era che perdeva

battiti sui rilievi cigliari la

sonnolenza adimensionale

nell'etere

che tutta s'ibridava e si nutriva di pulviscolari

assenze

'Riquadri clorati', 'etere' e 'pulviscoli' trovano posto in un mondo atomizzato, in cui si osservano particelle di materia immerse in un cosmo

${ }^{11}$ Come per Contropar(ab)ola, si citerà dalle prime edizioni di Per specula aenigmatis e Cumae, segnalando tra parentesi il titolo del testo e il corrispondente numero di pagina. 
di motivi ricorrenti, come quelli navali o floreali, lo stesso che permea Senza risposta (37):

Una cellula cos'è un gamete
fino a quale sponda si spinge la risacca
un cielo non più cielo chi mai l'abita
sono spinate rose o sono navi
insensate che s'inacquano
un atomo cos'è la luce
da quando stanno in agitazione
i matti del villaggio
ha ragione l'ossigeno o il nitrile
i rami sono andati giù chi li raccoglie
un attimo cos'è chi l'ha tagliata
quella pianta chi ha imposto il black-out
non c'ero io ma la cellula dov'è
il gamete chi l'ha strozzato chi:
s'inacqua ormai la mente allumacata.

Si può forse osservare che, in questa nuova fase, la terminologia desunta dalle scienze perde la sua etichetta di specialismo, per amalgamarsi del tutto con la medietas che è tratto dominante - a più riprese riconosciuto dai critici ${ }^{12}$ - della migliore poesia in italiano di Sovente. In effetti, da Cumae in avanti, le escursioni lessicali sono molto più contenute e in generale il ricorso agli scientismi si dirada, acquistando in compenso di consapevolezza. L'utilizzo di termini di provenienza fisica o astronomica, come quelli poc'anzi menzionati, svolge un ruolo speculare a quello del latino e del cappellese, che in qualità di lingue paritarie mettono in risalto aspetti diversi di una figura o una scena, non immediatamente percepibili nel testo italiano. I tecnicismi concorrono adesso alla raffigurazione di uno spazio 'altro' in cui trovano posto ectoplasmi e ombre, cioè elementi presenti ma intangibili, esattamente come gli 'atomi' e le 'nebulose' degli scenari soventiani.

${ }^{12}$ Cfr. De Blasi (2013: 98-99), dove l'italiano di Sovente viene definito "duttile e vivo, non selezionato dal poeta nei suoi registri letterari, ma accolto come lingua della comunicazione colloquiale, dove la colloquialità non conduce a una sua possibile consunzione, ma ad una raffinata e pacata essenzialità". 


\section{BIBLIOGRAFIA}

Alfano, G. (2010). Sovente, o lo spettro del paesaggio. Paragone-Letteratura, 87-88-89, 5-23.

Altieri Biagi, M. L. (1990). L'avventura della mente. Napoli: Morano.

Anceschi, L. (1976). Pascoli verso il Novecento. In R. Barilli e A. Guglielmi (a cura di), Gruppo 63. Critica e teoria (pp. 35-53). Milano: Feltrinelli.

Bozzola, S. (2014). La crisi della lingua poetica tradizionale. In G. Antonelli, M. Motolese \& L, Tomasin (a cura di), Storia dell'italiano scritto. Poesia (pp. 353-402). Roma: Carocci.

Bozzola, S. (2016). L'autunno della tradizione. La forma poetica dell'Ottocento. Firenze: Franco Cesati.

De Blasi, N. (2003). Le tre lingue poetiche di Michele Sovente. Poesia. Mensile internazionale di cultura poetica, 170, 52-55.

De Blasi, N. (2013). Le parole ritrovate nella poesia di Michele Sovente. Istmi, 31-32, 90-103.

Giudici, G. (1991). Poesie 1953-1990. Milano: Garzanti.

Giudici, G. (1993). Quanto spera di campare Giovanni. Milano: Garzanti.

Giudici, G. (1996). Empie stelle. Milano: Garzanti.

Grasso, M. (2012). Il Territorio dei Versi. Le ragioni della poesia di Michele Sovente. Napoli: Il Laboratorio / le edizioni.

Istmi (2013). Istmi, 31-32, 89-143.

Liberti, G. A. (2018). La Campania phoenix di Michele Sovente. Italica, 95, 2, 227-240.

Mengaldo, P. V. (1994). Storia della lingua italiana. Il Novecento. Bologna: il Mulino.

Montale, E. (2013). Ossi di seppia, a cura di P. Cataldi e F. D’Amely. Milano: Mondadori.

NDMSL (1976). Nuovissimo Dizionario Medico Saie / Larousse, a cura di A. Bairati, M.G. Malsani \& R. Valente, $5^{\text {a }}$ edizione. Torino: SAIE.

Ortore, M. (2012). Recensione di Casadei, A. (2011). Le sostanze (Borgomanero: Atelier). Atelier, XVII, 68, 108-113.

Ortore, M. (2016). La linea lucreziana nella poesia contemporanea. Il caso di Bruno Galluccio. In S. Redaelli (a cura di), La scienza nella letteratura italiana (pp. 171-182). Roma: Aracne.

Pagliarani, E. (2006). Tutte le poesie (1946-2005), a cura di A. Cortellessa. Milano: Garzanti.

Porro, M. (1973). I linguaggi della scienza e della tecnica. In G. L. Beccaria (a cura di), I linguaggi settoriali in Italia (pp. 181-206). Milano: Bompiani.

Serianni, L. (1989). Saggi di storia linguistica italiana. Napoli: Morano. 
Sovente, M. (1978). L'uomo al naturale. Firenze: Nuovedizioni Enrico Vallecchi.

Sovente, M. (1981). Contropar(ab)ola. Firenze: Nuovedizioni Enrico Vallecchi.

Sovente, M. (1990). Per specula aenigmatis. Milano: Garzanti.

Sovente, M. (1998). Cumae. Venezia: Marsilio.

Sovente, M. (2002). Carbones. Milano: Garzanti.

Sovente, M. (2005). Carta e formiche. Napoli: Centro di Cultura Contemporanea Napolic'è.

Sovente, M. (2008). Bradisismo. Milano: Garzanti.

Sovente, M. (2009). Superstiti. Genova: San Marco dei Giustiniani.

Sovente, M. (2019). Cumae, edizione critica e commentata a cura di Giuseppe Andrea Liberti. Macerata: Quodlibet.

Testa, E. (1999). Per interposta persona. Lingua e poesia nel secondo Novecento. Roma: Bulzoni.

Testa, E. (2005). Dopo la lirica. Poeti italiani 1960-2000. Torino: Einaudi.

Zublena, P. (2002). L'inquietante simmetria della lingua. Il linguaggio tecnico-scientifico nella narrativa italiana del Novecento. Alessandria: Edizioni dell'Orso.

Zublena, P. (2014). Dopo la lirica. In G. Antonelli, M. Motolese \& L. Tomasin (a cura di), Storia dell'italiano scritto. Poesia (pp. 403-452). Roma: Carocci.

\section{MICHELE SOVENTE AND THE WORDS OF SCIENCE. NOTES ON THE LEXICON OF CONTROPAR(AB)OLA}

\section{Summary}

After a brief recognition of science's role in contemporary Italian poetry, the paper intends to investigate a specific case of medical-scientific lexicon used in poems. Michele Sovente (1948-2011), known as a "trilinguistic" author due to his tendency to write texts in Italian, Latin and Cappella's dialect, began his career using exclusively Italian language. Nevertheless, Sovente's first books are full of medical and scientific terms that prove an interest for linguistic experimentations. This technical vocabulary is particularly remarkable in Contropar(ab)ola, Sovente's second book, published in 1981. In Contropar(ab)ola's poems, readers may find medical words like 'gerovital', 'gingivitis' or 'hemoptysis', but also terms from chemistry ('nitrile'), biology ('ozone', 'plantigrades') and other scientific areas. The study of these words could be helpful to understand their employment as metaphors or respecting their effective meaning, in order to show how scientific expressions can be used in different thematic contexts.

Key words: Michele Sovente, poetry, science, poetic language, sperimentalism, 20th century poetry. 